\title{
Small interfering RNA-mediated knockdown of fatty acid synthase attenuates the proliferation and metastasis of human gastric cancer cells via the $\mathrm{mTOR} / \mathrm{Gli1}$ signaling pathway
}

\author{
LIANG SUN $^{1}$, YIZHOU YAO ${ }^{1}$, GUOFENG PAN $^{1}$, SHENGHUA ZHAN ${ }^{2}$, WEIQIANG SHI ${ }^{2}$, TING LU ${ }^{3}$, \\ JINFENG YUAN ${ }^{1}$, KANGJUN TIAN ${ }^{1}$, LINHUA JIANG ${ }^{1}$, SHIDUO SONG ${ }^{1}$, XINGUO ZHU ${ }^{1}$ and SONGBING HE $^{1}$ \\ Departments of ${ }^{1}$ General Surgery, ${ }^{2}$ Pathology and ${ }^{3}$ Gastroenterology, \\ The First Affiliated Hospital of Soochow University, Suzhou, Jiangsu 215006, P.R. China
}

Received October 2, 2017; Accepted March 7, 2018

DOI: $10.3892 / \mathrm{ol} .2018 .8648$

\begin{abstract}
Fatty acid synthase (FASN), the main enzyme involved in de novo lipogenesis, is overexpressed in several types of tumor tissues. In addition, it is associated with tumor cell proliferation, metastasis, epithelial-mesenchymal transition (EMT) and a poor prognosis. However, the precise functions and internal mechanisms of FASN with regard to the proliferation, metastasis and EMT in gastric cancer (GC) cells remain elusive. The present study investigated FASN protein expression in 18 randomly selected pairs of $\mathrm{GC}$ tumors and matched normal tissues by western blot analysis. FASN-specific small interfering RNA (siRNA) was then transfected into SGC-7901 cells to examine the effect of FASN on proliferation and migration in vitro. Western blotting was used to detect the protein expression of FASN, EMT-related markers and key signaling molecules of the mechanistic target of rapamycin/zinc finger protein GLI1 (mTOR/Gli1) pathway. Reverse transcription-quantitative polymerase chain reaction was conducted to detect the mRNA expression of FASN and EMT-related markers. The FASN level was higher in the GC tissues compared with that in the surrounding normal tissues. Knockdown of FASN suppressed GC cell proliferation and metastasis in vitro. The silencing of FASN expression using siRNA reversed EMT at the protein and mRNA levels and decreased the expression of Gli1 via regulation of AMP-activated protein kinase/mTOR and protein kinase B/mTOR signaling in GC cells. Inhibition of FASN suppresses GC proliferation and metastasis through targeting
\end{abstract}

Correspondence to: Dr Songbing He or Dr Xinguo Zhu, Department of General Surgery, The First Affiliated Hospital of Soochow University, 188 Shizi Street, Suzhou, Jiangsu 215006, P.R. China

E-mail: captain_hsb@163.com

E-mail:zxg45@hotmail.com

Key words: fatty acid synthase, metastasis, epithelial-mesenchymal transition, gastric cancer of the mTOR/Gli1 signaling pathway, indicating that it may serve as a potential target for the treatment of GC.

\section{Introduction}

Gastric cancer (GC) is the fourth most common cancer and the second leading cause of cancer-associated mortality worldwide, with $>950,000$ newly diagnosed cases every year and an estimated 720,000 cases of GC-associated mortality in 2012 (1-3). Despite the development of surgical techniques and chemotherapy, the 5-year survival rate remains low, as the majority of GCs are diagnosed at advanced or metastatic stages $(4,5)$. Therefore, it is of great clinical importance to establish specific and sensitive biomarkers for the early diagnosis of GC and to identify effective therapeutic targets to prevent the metastasis of GC.

Several studies have demonstrated that elevated lipogenesis is associated with a poor prognosis in a number of cancer types including ovarian cancer, breast cancer and prostate cancer (6-8), and that it is involved in signal transduction of several types of tumors, including non-small cell lung cancer, prostate cancer and hepatocellular cancer (9-11). Fatty acid synthase (FASN), the main enzyme involved in de novo lipogenesis, is overexpressed in several types of tumor tissues including breast cancer, colorectal cancer and gastric cancer, and its overexpression is significantly associated with tumor cell proliferation, metastasis, epithelial-mesenchymal transition (EMT) and a poor prognosis (12-15). However, there are only a few studies focusing on FASN in GC (15-17), and these indicate that FASN is overexpressed in the GC tissues and blood serum of patients with GC, and that its overexpression is connected with poor survival rate. These data suggest that FASN serves a critical role in the development and progression of GC, but the precise functions and internal mechanisms of FASN in GC cell proliferation and metastasis remain elusive.

Available data indicate that the EMT serves a crucial role in tumor cell metastasis and invasion, which is accompanied by upregulation of mesenchymal-associated genes, including Vimentin, and downregulation of epithelial-associated markers, including E-cadherin $(18,19)$. Several types of signaling pathways and molecules are involved in the regulation of EMT $(18,20)$, and one of the most important signaling pathways 
is the Hedgehog (Hh) pathway. Furthermore, there is strong evidence that the Hh pathway and its effector zinc finger protein GLI1 (Gli1), one of the glioma-associated oncogenes, serve crucial roles in the development and progression of GC (21-24).

The present study investigated the role of FASN in GC development and determined its effect on the regulation of the Hh signaling pathway effector Glil, which is strongly associated with cell proliferation, metastasis and EMT in GC (21-24). To the best of our knowledge, for the first time, the present in vitro studies identified that FASN functions as a novel regulator for Gli1 expression to mediate GC cell proliferation and metastasis, with potential implications for novel approaches to GC therapy.

\section{Materials and methods}

Human GC tissues and cell lines. A total of 18 paired human GC tissues and adjacent normal tissues were collected immediately from patients with GC (10 male and 8 female) with a median age of 64 years (range, 31-75 years), who underwent surgical resection between January 2014 to December 2015 at the First Affiliated Hospital of Soochow University (Suzhou, Jiangsu, China). Written informed consent was obtained from all patients in this study, which was approved by the Biomedical Research Ethics Committee of the First Affiliated Hospital of Soochow University. The experiments performed on human tissues were in compliance with the Helsinki Declaration. The human GC SGC-7901 and MGC-803 cell lines were purchased from the Cell Bank of the Chinese Academy of Sciences (Shanghai, China) and were cultured in Roswell Park Memorial Institute (RPMI) 1640 medium (Hyclone; GE Healthcare Life Sciences, Logan, UT, USA) containing 10\% fetal bovine serum (FBS; Gibco; Thermo Fisher Scientific, Inc., Waltham, MA, USA), 100 U/ml penicillin $\mathrm{G}$ sodium and $100 \mu \mathrm{g} / \mathrm{ml}$ streptomycin sulfate (Gibco; Thermo Fisher Scientific, Inc.). All cells were maintained at $37^{\circ} \mathrm{C}$ in a humidified atmosphere containing $5 \% \mathrm{CO}_{2}$.

Transfection of small interfering RNA (siRNA). The siRNA against FASN (specific target sequence, 5'-TACGACTACGGC CCTCATT-3') and a negative control siRNA (target sequence, 5'-TTCTCCGAACGTGTCACGTTT-3') were synthesized by GenePharma (Shanghai, China). GC SGC-7901 and MGC-803 cell lines were transfected with control or FASN siRNA at a final concentration of $20 \mathrm{nM}$ using Lipofectamine ${ }^{\mathrm{TM}}$ RNAiMax (Invitrogen; Thermo Fisher Scientific, Inc.) according to the manufacturer's protocols. The cells were harvested for further experiments at 48-72 $\mathrm{h}$ post-transfection.

Protein extraction and western blot analysis. Whole protein extracts of tissues or cell lines (SGC7901 and MGC803) were lysed in ice-cold RIPA lysis buffer containing cocktails of protease and phosphatase inhibitors (Sigma-Aldrich; Merck KGaA, Darmstadt, Germany) according to the manufacturer's protocols. Protein concentrations were determined using a BCA protein assay kit (Pierce; Thermo Fisher Scientific, Inc.) Total proteins $(10 \mu \mathrm{g})$ from each lysate were separated by $8 \%$ SDS-PAGE and transferred onto polyvinylidene difluoride membranes (Bio-Rad Laboratories, Inc., Hercules, CA, USA), and then blocked with 5\% skimmed milk in TBS/0.1\% Tween for $1 \mathrm{~h}$ at room temperature. The membranes were then probed with the indicated primary antibodies diluted using PBS at $4^{\circ} \mathrm{C}$ with gentle agitation overnight, and then incubated with horseradish peroxidase-conjugated secondary antibodies diluted using PBS (dilution, 1:5,000; OriGene Technologies, Inc., Rockville, MD, USA) for $1 \mathrm{~h}$ at room temperature. Next, the proteins were visualized using chemiluminescence kit (EMD Millipore, Billerica, MA, USA) and signals were quantified by ImageJ software (version 1.46; National Institutes of Health, Bethesda, MD, USA). Antibodies used in this study are listed in Table I.

$R N A$ extraction and reverse transcription-quantitative polymerase chain reaction ( $R T-q P C R)$. The mRNA expression of FASN, E-cadherin and Vimentin in SGC-7901 and MGC-803 cells transfected with negative control siRNA or siRNA against FASN was quantified by RT-qPCR. Total RNA was extracted from the cells using TRIzol reagent (Invitrogen; Thermo Fisher Scientific, Inc.) and cDNA was synthesized from $2 \mu \mathrm{g}$ RNA using the First Strand cDNA Synthesis kit (Fermentas; Thermo Fisher Scientific, Inc.) according to the manufacturer's protocols. RT-qPCR was performed using Power SYBR ${ }^{\circledR}$ Green PCR Master mix (Applied Biosystems; Thermo Fisher Scientific, Inc.) on the 7500 Real-Time PCR system (Applied Biosystems; Thermo Fisher Scientific, Inc.). The 18S rRNA was used as a loading control for each specific gene. The sequences for the sense (S) and antisense (AS) primers were as follows: Human-FASN-S, 5'-CTTCCGAGATTCCATCCT ACGC-3' and human-FASN-AS, 5'-TGGCAGTCAGGCTCA CAAACG-3'; human-E-cadherin-S, 5'-CGGGAATGCAGT TGAGGATC-3' and human-E-cadherin-AS, 5'-AGGATGGTG TAAGCGATGGC-3'; human-Vimentin-S, 5'-GAGAACTTT GCCGTTGAAGC-3' and human-Vimentin-AS, 5'-GCTTCC TGTAGGTGGCAATC-3'; and human-18S rRNA-S, 5'-GTA ACCCGTTGAACCCCATT-3' and human-18S rRNA-AS, 5'-CCATCCAATCGGTAGTAGCG-3'. The PCR conditions consisted of $5 \mathrm{~min}$ at $95^{\circ} \mathrm{C}$ for 1 cycle, followed by $30 \mathrm{sec}$ at $95^{\circ} \mathrm{C}, 30 \mathrm{sec}$ at $55^{\circ} \mathrm{C}, 30 \mathrm{sec}$ at $72^{\circ} \mathrm{C}$ and $7 \mathrm{~min}$ at $72^{\circ} \mathrm{C}$ for 40 cycles. The relative fold-changes in mRNA expression were calculated using the $2^{-\Delta \Delta \mathrm{Cq}}$ method (25), where the mean of the $\Delta \mathrm{Cq}$ values for the amplicon of interest was normalized to that of $18 \mathrm{~S}$ rRNA and compared with the control specimens.

MTT assay of cell proliferation. Cell proliferation was determined using an MTT assay kit (Amresco LLC, Solon, OH, USA). Following transfection, 4,000 cells were seeded in 96-well plates for 24, 48 and $72 \mathrm{~h}$, and then incubated with MTT solution-containing culture medium at $37^{\circ} \mathrm{C}$ for $4 \mathrm{~h}$. The supernatants were then removed and formazan crystals were dissolved in $150 \mu \mathrm{l}$ dimethyl sulfoxide. Following gentle agitation for $10 \mathrm{~min}$, the absorbance at $490 \mathrm{~nm}$ was measured using a microplate reader.

Colony formation assay. In total, 1,000 cells were placed in 6-well plates for 10 days, and then fixed with $4 \%$ paraformaldehyde (Beyotime Institute of Biotechnology; Haimen, China) for $10 \mathrm{~min}$ at room temperature and stained with $0.1 \%$ crystal violet (Beyotime Institute of Biotechnology) for $15 \mathrm{~min}$ at room temperature. The number of foci containing $>100$ cells was determined at $\mathrm{x} 40$ magnification using an optical microscope (Nikon Corporation; Tokyo, Japan), and the images were captured by a digital camera (Nikon Corporation). 
Table I. Antibody information.

\begin{tabular}{llcc}
\hline Name & \multicolumn{1}{c}{ Company } & Catalog no. & Concentration \\
\hline FASN & Cell Signaling Technology, Inc. & 3180 & $1: 1,000$ \\
E-cadherin & Cell Signaling Technology, Inc. & 3195 & $1: 500$ \\
Vimentin & Cell Signaling Technology, Inc. & 5741 & $1: 500$ \\
Phospho-AMPK $\alpha$ (Thr172) & Cell Signaling Technology, Inc. & 4535 & $1: 1,000$ \\
Phospho-Akt (Ser473) & Cell Signaling Technology & 4058 & $1: 1,000$ \\
Phospho-mTOR (S2448) & Cell Signaling Technology, Inc. & 9205 & $1: 1,000$ \\
Gli1 & Abcam & Ab15179 & $1: 2,000$ \\
GAPDH & Beyotime & AG019 & $1: 2,000$
\end{tabular}

Cell Signaling Technology, Inc., Danvers, MA, USA; Abcam, Cambridge, UK; Beyotime Institute of Biotechnology, Haimen, China. FASN, fatty acid synthase; phosphor, phosphorylated; AMPK $\alpha$, AMP-activated protein kinase $\alpha$; Akt, protein kinase B; mTOR, mechanistic target of rapamycin; Gli1, zinc finger protein GLI1.

A

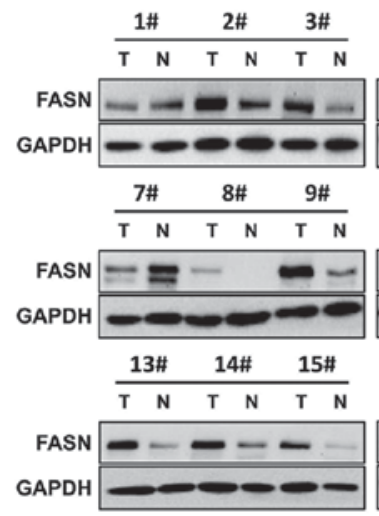

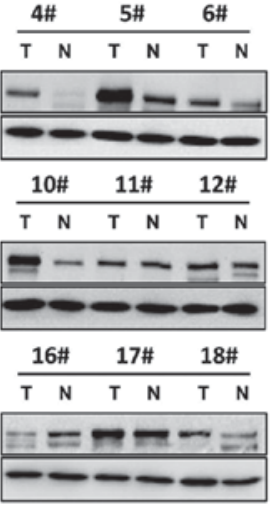

B

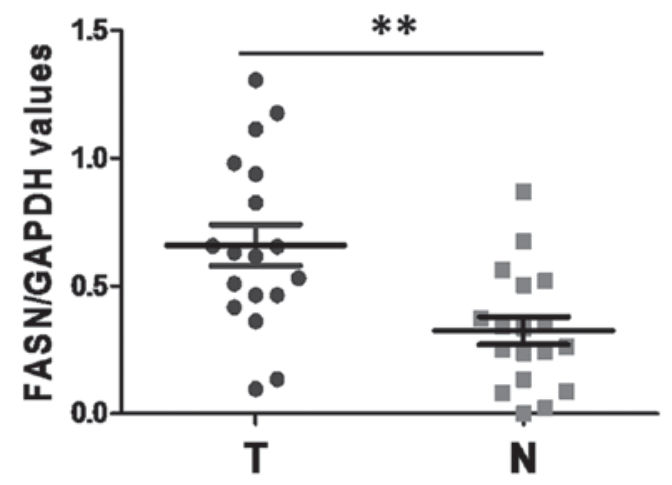

Figure 1. FASN is upregulated in GC. (A) Western blotting of FASN protein in GC tumors. In total, 18 randomly selected pairs of GC tumors and matched normal tissues are presented. GAPDH was used as a loading control. (B) Scatter plot analysis of FASN/GAPDH levels in 18 GC tumors and paired normal tissues. Data are presented as the mean \pm standard error of the mean $(n=18)$. Statistical significance was determined by a two-tailed, unpaired Student's t-test. ${ }^{* *} \mathrm{P}<0.01$. GC, gastric cancer; FASN, fatty acid synthase; T, tumors; N, matched normal tissues; GAPDH, glyceraldehyde 3-phosphate dehydrogenase.

Cell migration assay. GC cell migration was assessed using Transwell chambers (pore size, $8.0 \mu \mathrm{m}$; Corning Inc., Corning, New York, USA). The cells were re-suspended in serum-free RPMI medium, then cell suspensions (200 $\mu$ l containing 50,000 cells) were seeded onto the filters in 24-well chambers; $750 \mu \mathrm{l}$ medium containing 10\% FBS was placed in the lower chambers as a chemoattractant. The cells were allowed to migrate for $24 \mathrm{~h}$ at $37^{\circ} \mathrm{C}$. Cells remaining on the upper surface of the membrane were then removed using a cotton swab. The filters were fixed with $4 \%$ paraformaldehyde (Beyotime Institute of Biotechnology) for $10 \mathrm{~min}$ at room temperature, and the cells were stained with $0.1 \%$ crystal violet solution (Beyotime Institute of Biotechnology) for $15 \mathrm{~min}$ at room temperature. The cells that had migrated from the upper to the lower side of the filter were counted in 5 randomly selected fields per sample using a light microscope (magnification, x200; Nikon Corporation).

Statistical analysis. Data of MTT assay are presented as the standard deviation and the other are presented as mean \pm standard error. Statistical significance was analyzed using Student's t-test (unpaired, two-tailed) or one-way analysis of variance followed by the Student-Newman-Keuls test. $\mathrm{P}<0.05$ was considered to indicate a statistically significant difference. All statistical analyses were performed with SPSS17.0 software (SPSS, Inc., Chicago, IL, USA).

\section{Results}

FASN is overexpressed in human GC. Previous studies (15-17) have provided evidence that FASN is overexpressed in GC tissues and that its overexpression is closely associated with GC metastasis and patient survival. To further investigate the potential role of FASN in the development of GC, western blotting was used in the present study to examine FASN expression in human GC tissues and paired adjacent normal tissues. Notably, the results showed that abundant FASN expression was present in the primary GC tumors. By contrast, the expression of FASN was significantly lower in the matched paraneoplastic tissues (Fig. 1A and B). These data suggested that increased FASN expression may be associated with the development of GC. 
A

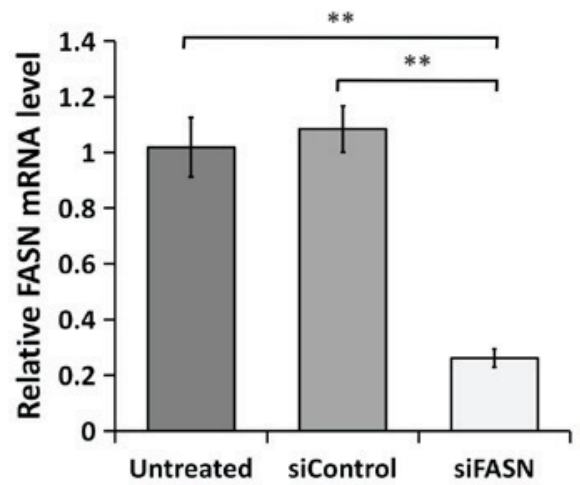

B

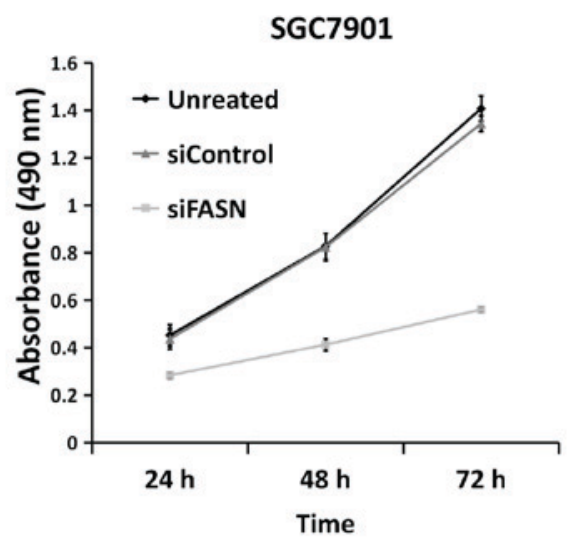

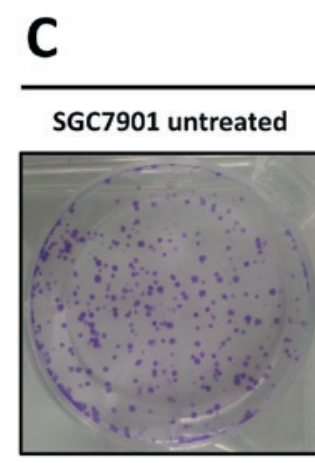

Colony formation

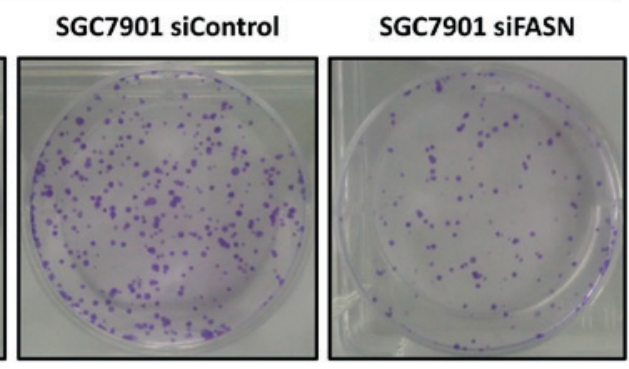

D

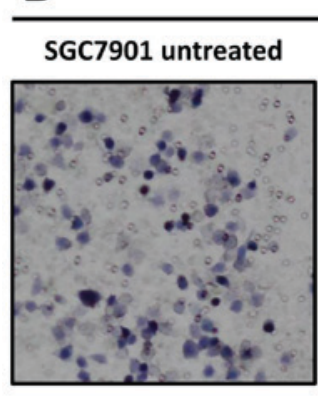

Migration

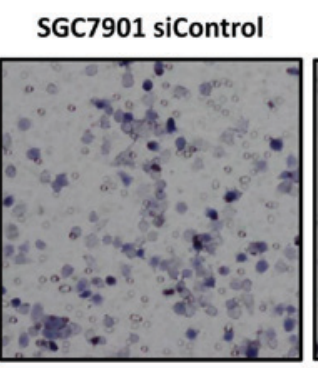

SGC7901 SIFASN

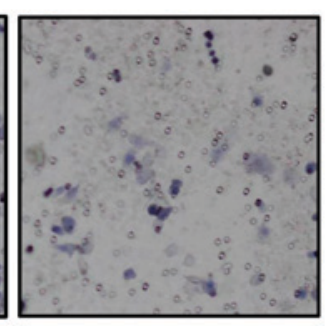

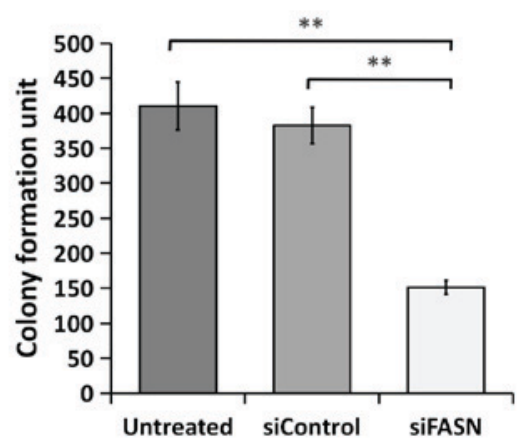

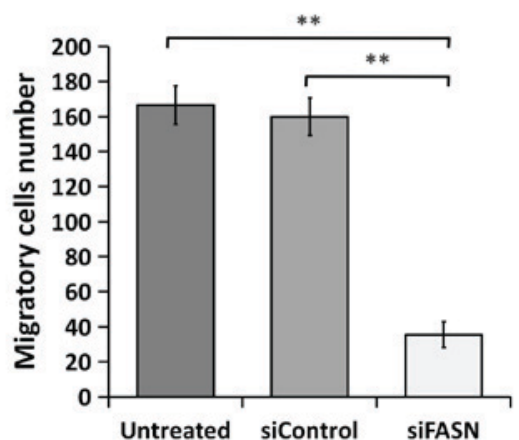

Figure 2. Knockdown of FASN by siRNA inhibits gastric cancer cell proliferation and metastasis in vitro. (A) Reverse transcription-quantitative polymerase chain reaction analysis indicated that FASN expression was strongly decreased in FASN-depleted SGC-7901 cells at the mRNA level. 18S rRNA was used as a loading control. (B) Downregulation of FASN expression inhibited the proliferation of SGC-7901 cells compared with that in the untreated cells and control siRNA-transfected cells, as determined by MTT assay. Figures are curves of SGC-7901 cell proliferation following transfection for 24,48 and $72 \mathrm{~h}$ for MTT assays. Data are presented as the mean \pm standard deviation $(n=3)$. (C) SGC-7901 cells transfected with control siRNA or siRNA against FASN were maintained in culture medium for 10 days and then fixed and stained with $0.1 \%$ crystal violet, and the colonies containing $>100$ cells were counted manually. The representative images are presented (left; magnification, $\mathrm{x} 1$ ), and the relative number of colonies was counted (right). The bands were quantified and presented as the mean \pm SEM of three independent experiments. (D) Migration assays were performed in wild-type SGC-7901 cells and in cells transfected with negative control siRNA or siRNA against FASN. Representative images are presented (left; magnification, x200) and the relative numbers of migratory cells (right) were counted. The bands were quantified and presented as the mean \pm SEM of three independent experiments. Statistical significance was determined by one-way analysis of variance. ${ }^{* *} \mathrm{P}<0.01 ;{ }^{* * *} \mathrm{P}<0.001$. FASN, fatty acid synthase; siRNA, small interfering RNA; SEM, standard error of the mean; siControl, control siRNA; siFASN, siRNA against FASN; untreated, wild-type cells.

FASN deficiency inhibits GC cell proliferation and metastasis in vitro. Since FASN expression is upregulated in GC tissues, the present study assessed whether FASN has a causal role in regulating GC cell phenotypes. First, FASN expression was knocked down using FASN-specific siRNA in the GC SGC-7901 cell line. Furthermore, the knockdown efficiency was confirmed by RT-qPCR (Fig. 2A). In order to determine the effect of FASN on the proliferation of SGC-7901 cells in vitro, the proliferation curves were detected by MTT assays at 24, 48 and $72 \mathrm{~h}$ following $48 \mathrm{~h}$ of transfection (Fig. 2B).
It was found that the SGC-7901 cells transfected with siRNA against FASN experienced significant inhibition of cell proliferation compared with the SGC-7901 cells transfected with control siRNA or the wild-type untreated SGC-7901 cells. In agreement with this result, colony formation assays also revealed that the ability of SGC-7901 cells with downregulated FASN to form foci displayed an apparent reduction compared with that of the control-siRNA and untreated cells, respectively (Fig. 2C). In a Transwell migration assay, the migration ability of FASN-depleted SGC-7901 cells was greatly inhibited 


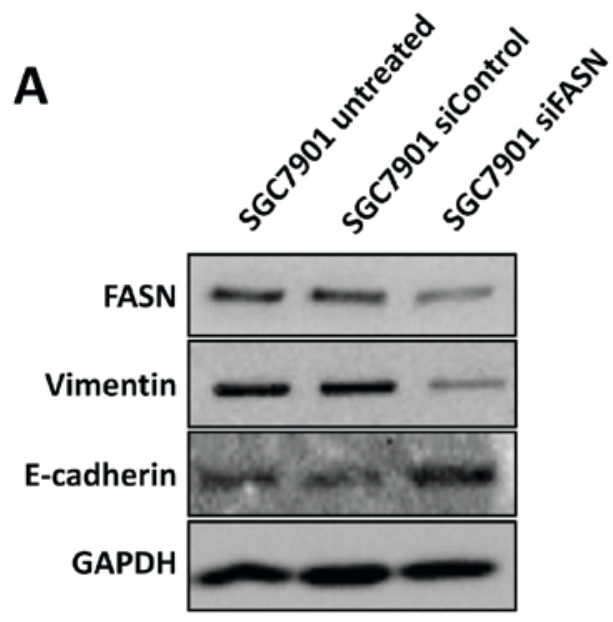

B

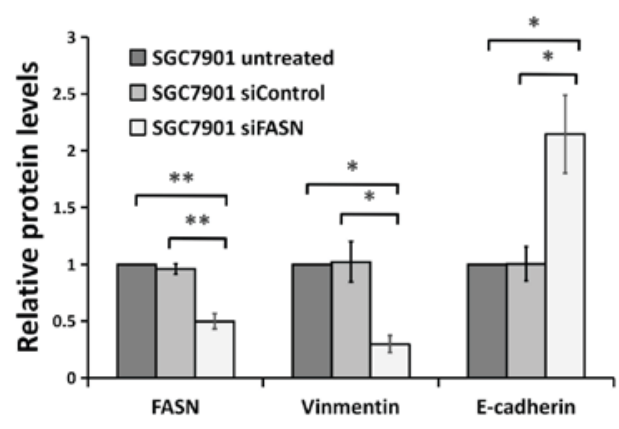

C

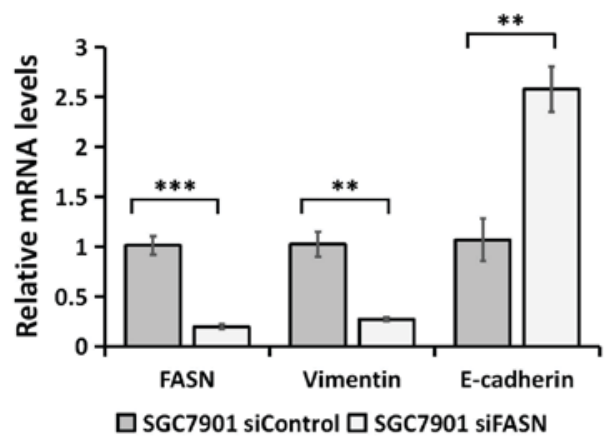

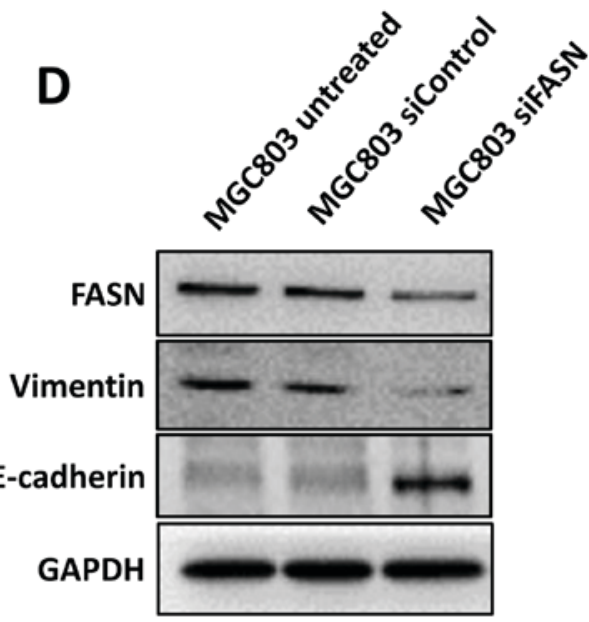

E

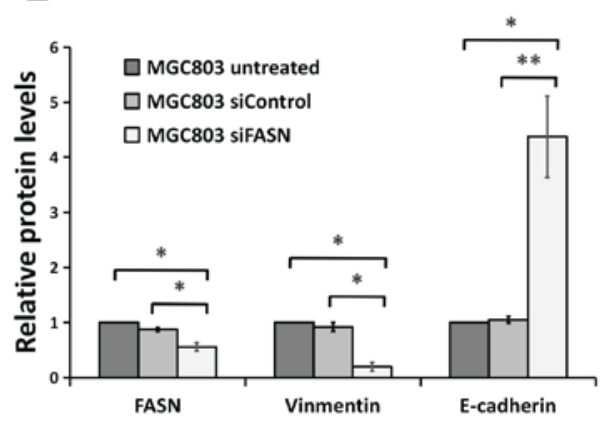

$\mathbf{F}$

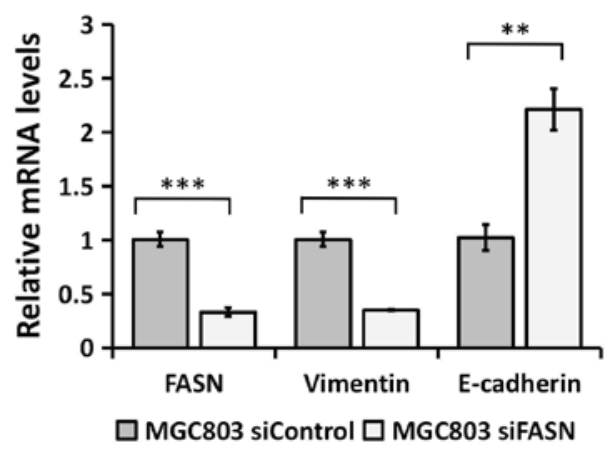

Figure 3. Knockdown of FASN expression by siRNA reversed EMT at the protein and mRNA levels in gastric cancer cells. (A) Western blot analysis of the Vimentin and E-cadherin expression in wild-type SGC-7901 cells and in cells transfected with negative control siRNA or siRNA against FASN. GAPDH was used as a loading control. (B) The bands were quantified and presented as the mean \pm SEM of three independent experiments. (C) RT-qPCR analysis of Vimentin and E-cadherin expression in SGC-7901 cells transfected with negative control siRNA or siRNA against FASN. The bands were presented as the mean \pm SEM $(\mathrm{n}=4)$. 18S rRNA was used as a loading control. (D) Western blotting analysis of the Vimentin and E-cadherin expression in wild-type MGC-803 cells and in cells transfected with negative control siRNA or siRNA against FASN. GAPDH was used as a loading control. (E) The bands were quantified and presented as the mean \pm SEM of three independent experiments. (F) RT-qPCR analysis of Vimentin and E-cadherin expression in MGC-803 cells transfected with negative control siRNA or siRNA against FASN. The bands were presented as the mean $\pm \operatorname{SEM}(n=4)$. $18 \mathrm{~S}$ rRNA was used as a loading control. Statistical significance was determined by Student's t-test (unpaired, two-tailed) or one-way analysis of variance. ${ }^{*} \mathrm{P}<0.05 ;{ }^{* * *} \mathrm{P}<0.01 ;{ }^{* * * *} \mathrm{P}<0.001$. RT-qPCR, reverse transcription-quantitative polymerase chain reaction; FASN, fatty acid synthase; siRNA, small interfering RNA; SEM, standard error of the mean; siControl, control siRNA; siFASN, siRNA against FASN; untreated, wild-type cells; EMT, epithelial-mesenchymal transition; GAPDH, glyceraldehyde 3-phosphate dehydrogenase.

compared with that of the control-siRNA and untreated cells (Fig. 2D). These results indicated that the knockdown of FASN by siRNA inhibits GC cell proliferation and metastasis in vitro.

Silencing FASN expression by siRNA regulates EMT in GC cells. Since FASN is involved in GC metastasis, it is possible that FASN may regulate EMT, which is an early event in the metastasis of cancer $(18,19)$. To test this, the expression of the epithelial marker E-cadherin and the mesenchymal marker
Vimentin was analyzed using western blotting and RT-qPCR assays. The western blotting analysis revealed that cells transfected with siRNA against FASN exhibited a significant decrease in Vimentin protein expression compared with cells transfected with control siRNA and the wild-type untreated cells, as well as a significant increase in E-cadherin protein expression in the SGC-7901 (Fig. 3A and B) and MGC-803 (Fig. 3D and E) GC cell lines. Consistent with the western blot analysis, the downregulation of FASN expression significantly 

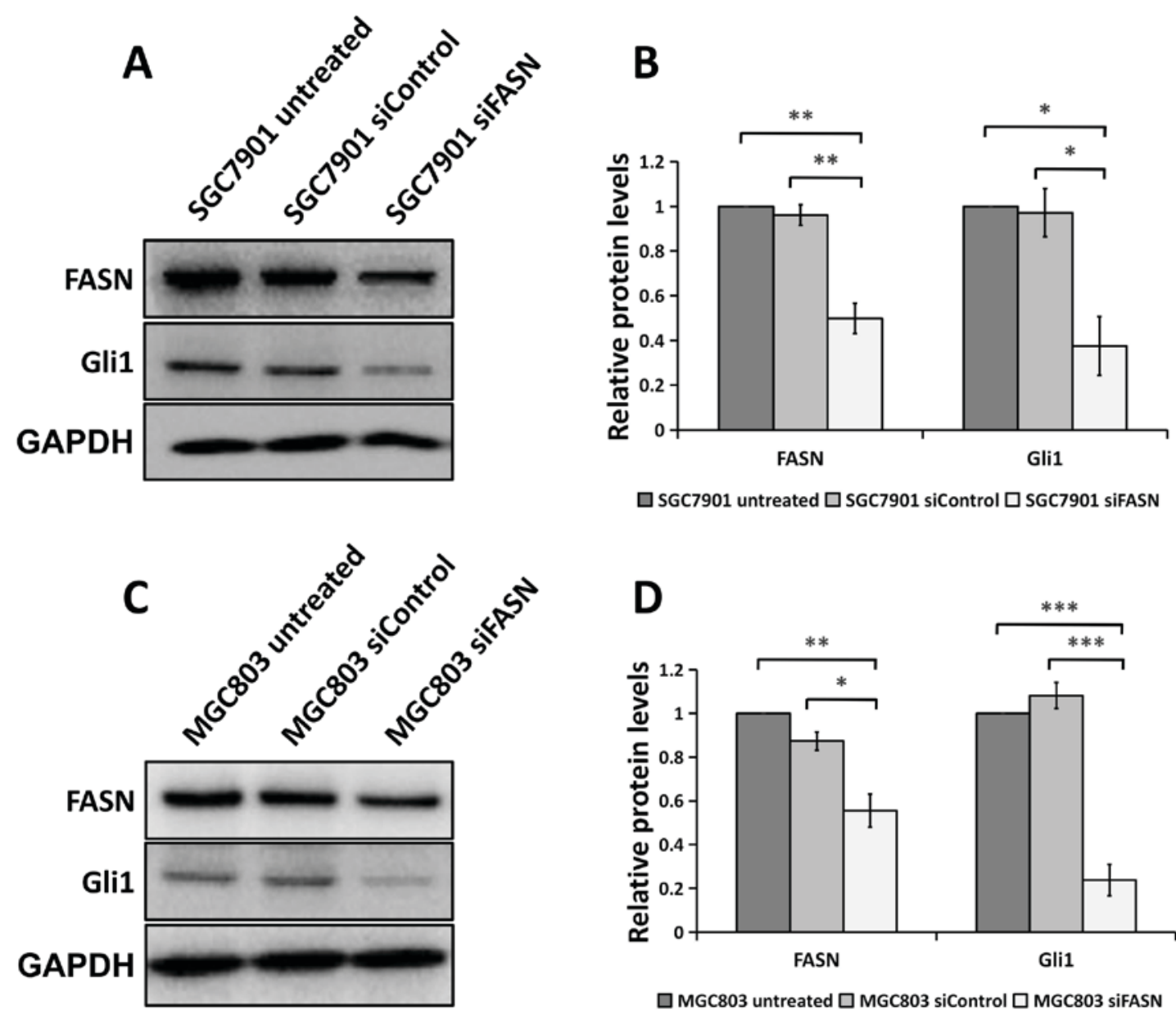

口SGC7901 untreated $\square$ SGC7901 siControl $\square$ SGC7901 SiFASN

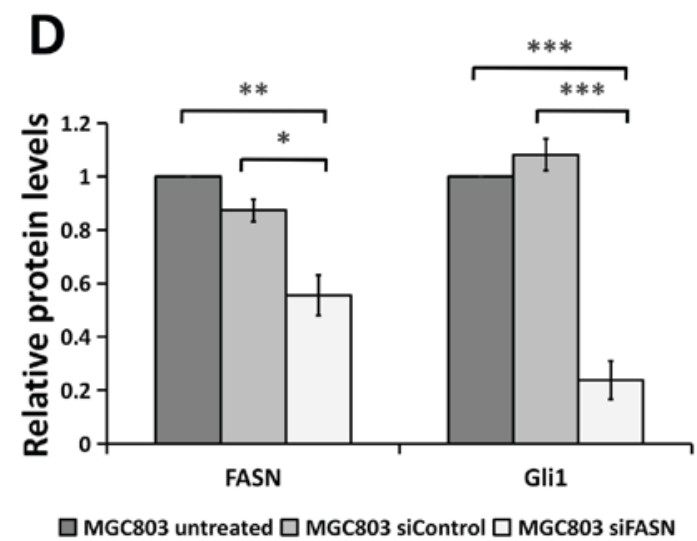

Figure 4. FASN regulates Gli1 expression in gastric cancer cells. (A) Western blotting analysis of the Gli1 expression in wild-type SGC-7901 cells and in cells transfected with negative control siRNA or siRNA against FASN. GAPDH was used as a loading control. (B) The bands were quantified and presented as the mean \pm SEM of three independent experiments. (C) Western blotting analysis of and Gli1 expression in wild-type MGC-803 cells and in cells transfected with negative control siRNA or siRNA against FASN. GAPDH was used as a loading control. (D) The bands were quantified and presented as the mean \pm SEM of three independent experiments. Statistical significance was determined by one-way analysis of variance. FASN, fatty acid synthase; siRNA, small interfering RNA; SEM, standard error of the mean; siControl, control siRNA; siFASN, siRNA against FASN; untreated, wild-type cells; Gli1, zinc finger protein GLI1; GAPDH, glyceraldehyde 3-phosphate dehydrogenase.

enhanced E-cadherin gene expression and attenuated Vimentin gene expression at the mRNA level in the SGC-7901 (Fig. 3C) and MGC-803 (Fig. 3F) GC cell lines. All these data indicated that FASN exerts a crucial role in modulating EMT in GC cells.

FASN loss decreases Gli1 level in GC cells. Since aberrant Gli1 expression in the Hh pathway underlies the development and metastasis of cancer $(18,20,21)$, it was assessed whether FASN serves any role in modulating Gli1 expression with regard to GC development and progression. Unexpectedly, as shown in Fig. 4A and B, cells transfected with siRNA against FASN exhibited a significant decrease in Glil protein expression compared with cells transfected with control siRNA or the wild-type untreated cells, indicating that FASN may regulate Gli1 expression in SGC-7901 and MGC-803 GC cells.

FASN modulates Glil expression through regulating the mTOR signaling pathway in GC cells. As aforementioned, the blockade of FASN attenuated Gli1 protein expression in GC cells. Next, the present study determined how FASN regulates Gli1 expression in GC. A range of recent findings have shown that FASN can stimulate mTOR signaling and that by contrast, silencing FASN impairs mTOR signaling in ovarian cancer (26-28). Furthermore, it has been reported that activated mTOR can promote Gli1 transcriptional activity and oncogenic function through ribosomal protein S6 kinase $\beta 1$ (S6K1)-mediated Gli1 phosphorylation in esophageal adenocarcinoma (23). The present study investigated whether FASN modulates Gli1 expression via regulating mTOR signaling in GC. As expected, the blockade of FASN by siRNA in the SGC-7901 and MGC-803 cells greatly decreased phosphorylation of mTOR while mTOR expression itself has not changed, which indicated that knockdown of FASN suppressed the activation of mTOR (Fig. 5A and B), indicating that FASN modulates Gli1 expression probably via its regulation of the mTOR signaling pathway in GC cells. These data suggested that FASN modulates the proliferation and metastasis of GC potentially via regulation of the mTOR/Gli1 signaling pathway.

FASN regulates AMP-activated protein kinase (AMPK) and protein kinase $B(A K T)$ in $G C$ cells. Available data suggest that blockade of FASN activates the mTOR repressor AMPK $\alpha$ causing the mTOR signaling inhibition in ovarian cancer (28), 


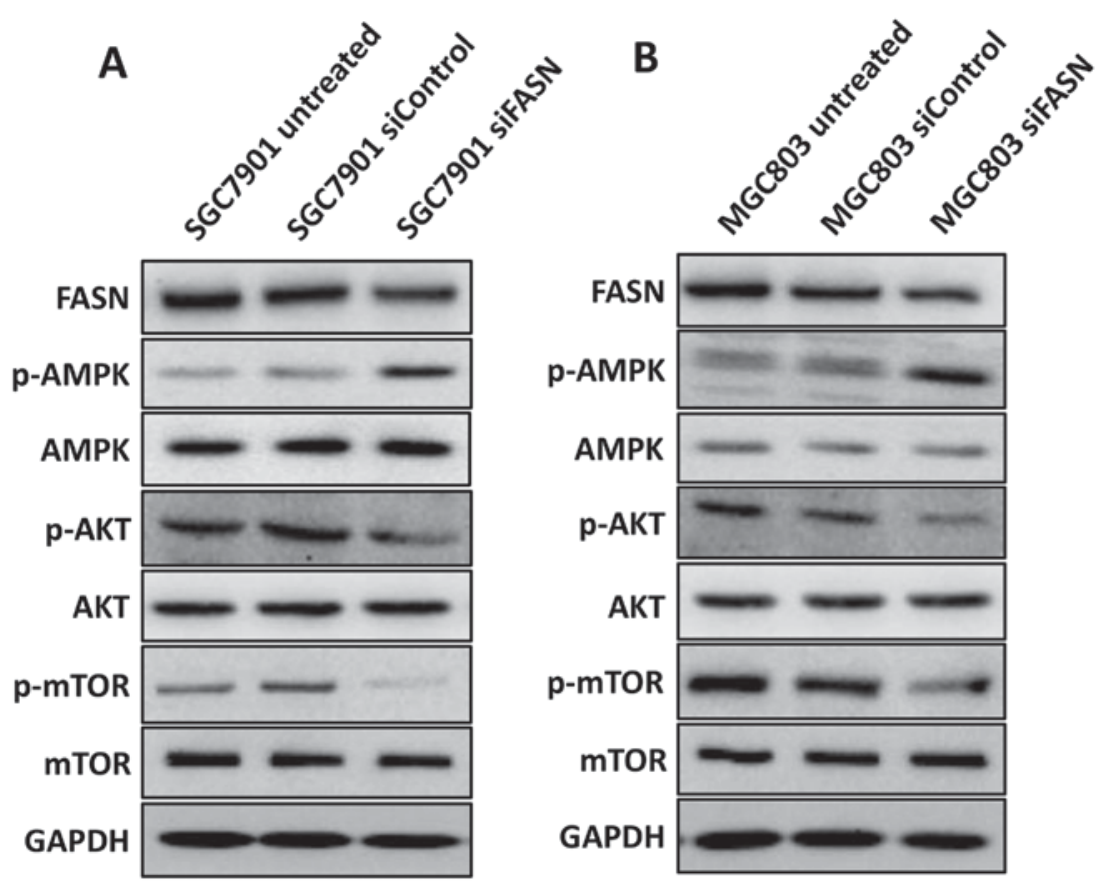

Figure 5. FASN modulates the activation of AMPK, AKT and mTOR in gastric cancer cells. (A) Western blotting analysis of the p-AMPK, p-AKT and p-mTOR expression in wild-type SGC-7901 cells and in cells transfected with negative control siRNA or siRNA against FASN. GAPDH was used as a loading control. (B) Western blotting analysis of the p-AMPK, p-AKT and p-mTOR expression in wild-type MGC-803 cells and in cells transfected with negative control siRNA or siRNA against FASN. GAPDH was used as a loading control. FASN, fatty acid synthase; siRNA, small interfering RNA; siControl, control siRNA; siFASN, siRNA against FASN; untreated, wild-type cells; AMPK, AMP-activated protein kinase; AKT, protein kinase B; mTOR, mechanistic target of rapamycin; p-, phosphorylated; GAPDH, glyceraldehyde 3-phosphate dehydrogenase.

and FASN inhibition can also inactivate the activity of AKT in various types of cancer (29-31). Since the activation of AMPK and AKT serves a vital role in regulating mTOR activity in cancer (32), the present study assessed whether AMPK and AKT are involved in the regulation of FASN-mediated mTOR/Gli1 activation in GC. Unexpectedly, as shown in Fig. 5A and B, silencing FASN markedly increased phosphorylation of AMPK and attenuated phosphorylation of AKT while total AMPK or AKT expression has not changed in the SGC-7901 and MGC-803 cell lines, indicating that FASN modulates mTOR activation, probably via its regulation of AMPK and AKT in GC cells.

\section{Discussion}

Fatty acid metabolism serves a crucial role in carcinogenesis and is strongly involved in the signal transduction of several types of tumor cells (9-11). FASN is a key enzyme of de novo fatty acid synthesis, which supplies lipids for membrane production. In cancer cells, FASN is commonly overexpressed, providing cancer cells with an extra source of cellular fatty acids, and is significantly associated with tumor cell proliferation, metastasis and a poor prognosis (12-15). Inhibition of tumor FASN activity attenuates tumor cell proliferation and metastasis, and induces apoptosis in vitro and in vivo $(11,14,27,28,30,31)$, suggesting that FASN is an attractive target for cancer therapy.

However, studies focusing upon FASN in GC are limited. Previous studies (15-17) have provided evidence that FASN is overexpressed in GC tissues, and that its overexpression is closely associated with GC metastasis and survival, indicating that FASN contributes to the development and progression of
GC. However, the functional role of FASN expression in GC cells remains unclear, and the concrete molecular mechanisms of the regulation of cell proliferation and metastasis by FASN are not well understood.

The present study demonstrated that the expression of FASN in GC tissues was increased compared with that in adjacent normal tissues, as indicated by other previous studies $(15,16)$. To the best of our knowledge, the present study also provided the first evidence that the knockdown of FASN via siRNA inhibited the proliferation and migration of GC cells. In view of the important role of EMT in tumor metastasis during tumor progression $(18,19)$, the role of FASN on the EMT of GC cells was also investigated for the first time in the present study, and it was revealed that FASN can modulate the expression of EMT markers E-cadherin and Vimentin in GC SGC-7901 and MGC-803 cells.

Emerging literature suggests that the Hh pathway and its effector Glil are highly involved in the proliferation, metastasis and EMT of numerous types of malignant tumors, including GC (18,20-24). The Hh pathway includes the canonical and non-canonical signaling pathways (33). The Hh ligands binds and inactivates the $\mathrm{Hh}$ receptor, protein patched homolog 1 (PTCH1), leading to the release of the G-coupled receptor-like signal transducer Smoothened homolog (Smo), which then activates Gli by blocking their inhibitory partner, suppressor of fused homolog $(\mathrm{SuFu})$; this is the canonical Hh signaling pathway. Besides being activated by the $\mathrm{Hh}$ ligand-PTCH1-Smo axis, Gli proteins, mainly Gli1, can also be activated by other signaling molecules, including mTOR/S6K1 and mitogen-activated protein kinase/extracellular signal-regulated kinase $(33,34)$, which is termed non-canonical Hh signaling. 
The present study identified siRNA-mediated knockdown of FASN decreased Gli1 level in GC cells, suggesting that FASN may serve as a regulator of the Hh signaling pathway effector Gli1 in GC tumorigenesis and metastasis. Considering that FASN can stimulate mTOR signaling and FASN inhibition impairs this mTOR signaling $(26-28,31)$ and as activated mTOR can induce Gli1 transcriptional activity and oncogenic function (34), the present study assessed whether FASN modulates Gli1 expression via regulating the mTOR signaling in GC. In accordance with other studies (26-28,31), the blockade of FASN suppressed the phosphorylation of mTOR in GC cells (Fig. 5A and B), suggesting that FASN modulates the proliferation and metastasis of GC potentially via the regulation of the mTOR/Gli1 signaling pathway.

Recent studies suggest that the inhibition of FASN activates the mTOR repressor AMPK $\alpha$ (28) and suppresses the activity of AKT (29-31). Since the activation of AMPK and AKT serves a vital role in regulating mTOR activity in cancer (32), the present study next investigated whether AMPK and AKT are involved in the regulation of FASN-mediated mTOR/Gli1 activation in GC. Unexpectedly, the study showed that silencing FASN activated AMPK and attenuated phosphorylation of AKT in the GC SGC-7901 and MGC-803 cell lines, indicating that FASN modulates mTOR activation, at least in part, by the regulation of AMPK and AKT in GC cells.

Available data suggest that mTOR signaling upregulates FASN by the induction of sterol regulatory element binding protein-1 (35), and FASN in turn can stimulate mTOR signaling, with its blockade impairing mTOR signaling (26-28,31), implying the presence of positive feedback loops between FASN and mTOR signaling in cancer. The impact of mTOR signaling on FASN is already well characterized, but reverse actions from FASN towards mTOR remain elusive. A recent study revealed that AMPK links FASN-blockade to mTOR suppression and growth inhibition (28). In the present study, in GC cells, it was confirmed that FASN modulates mTOR activation via regulation of AMPK and AKT.

Extensive studies have demonstrated that $\mathrm{Hh}$ signaling triggers FASN expression in a Smo-dependent manner to regulate de novo lipid synthesis in progenitor cells and medulloblastoma $(36,37)$, but whether FASN serves any role in regulating the Hh pathway remains unclear. To the best of our knowledge, the present study provided the first evidence that siRNA-mediated FASN-knockdown decreased the expression of the Hh pathway effector Gli1, at least in part via regulation of AMPK/mTOR and AKT/mTOR signaling in GC cells, but whether FASN regulates Gli1 expression in a Smo-dependent manner was not elucidated and warrants future investigation.

There were certain limitations in the present study. Firstly, only western blot analysis was used to examine FASN expression in human GC tissues and paired adjacent normal tissues. The level of FASN could be analyzed by immunohistochemical staining and the association with clinicopathological parameters in GC patients could be assessed. Secondly, AMPK/mTOR activity or Gli1 expression was not detected in the GC and paired normal tissues, nor was the effect of the depletion of FASN on GC tumorigenesis and metastasis measured in vivo. Thirdly, multi-level suppression of FASN or FASN overexpression in GC cells was not performed and siRNA was only used against FASN to inhibit
FASN expression. The mechanism of FASN regulation of AMPK and AKT in GC will be investigated in a future study. Further research should be undertaken to further investigate the function of FASN in GC. Taken together, the results of the current study indicate that the knockdown of FASN by siRNA suppresses GC proliferation and metastasis through targeting mTOR/Gli1 signaling, indicating that it may serve as a potential target for the treatment of GC.

In summary, the present in vitro experiments not only indicated that FASN functions as a novel regulator of GC cell proliferation and metastasis, but also emphasized the potential role of FASN in regulating Glil expression. Moreover, a critical mechanism was revealed for FASN in the regulation of GC tumorigenesis and metastasis through its participation in the non-classical Hh signaling pathway. This may highlight a novel entry point for treating GC by targeting the FASN/mTOR/Gli1 signaling axis.

\section{Acknowledgements}

Not applicable.

\section{Funding}

This study was supported by the Project of Nature Science Foundation of China (grant no. 81672348), the National Science Foundation of Jiangsu Province, China (grant no. BK2016255), the Six Major Talent Peak Project of Jiangsu Province, China (grant no. 2015-WSW-014), the Six One Project for Advanced Medical Talent of Jiangsu Province, China (grant no. LGY2016031) and the Jiangsu Provincial Medical Youth Talent (grant no. QNRC2016735).

\section{Availability of data and materials}

The datasets used and/or analyzed during the present study are available from the corresponding author on reasonable request.

\section{Authors' contributions}

LS and SH conducted the research, analyzed the data and wrote the manuscript. YY, GP, SZ, WS, TL, JY, KT, LJ and SS contributed to data collection and analysis. LS, XZ and SH designed the study and supervised the manuscript.

\section{Ethics approval and consent to participate}

The study was approved by the Biomedical Research Ethics Committee of the First Affiliated Hospital of Soochow University. The experiments performed on human tissues were in compliance with the Helsinki Declaration.

\section{Consent for publication}

Written informed consent for publication was obtained from all patients in this study.

\section{Competing interests}

The authors declare that they have no competing interests. 


\section{References}

1. Van Cutsem E, Sagaert X, Topal B, Haustermans K and Prenen H: Gastric cancer. Lancet 388: 2654-2664, 2016.

2. Ferlay J, Steliarova-Foucher E, Lortet-Tieulent J, Rosso S, Coebergh JW, Comber H, Forman D and Bray F: Cancer incidence and mortality patterns in Europe: Estimates for 40 countries in 2012. Eur J Cancer 49: 1374-1403, 2013.

3. Gores GJ and Lieberman D: Good news-bad news: Current status of GI cancers. Gastroenterology 151: 13-16, 2016.

4. Ferlay J, Soerjomataram I, Dikshit R, Eser S, Mathers C, Rebelo M, Parkin DM, Forman D and Bray F: Cancer incidence and mortality worldwide: Sources, methods and major patterns in GLOBOCAN 2012. Int J Cancer 136: E359-E386, 2015.

5. Shridhar R, Almhanna K, Hoffe SE, Fulp W, Weber J, Chuong MD and Meredith KL: Increased survival associated with surgery and radiation therapy in metastatic gastric cancer: A surveillance, epidemiology, and end results database analysis. Cancer 119: 1636-1642, 2013

6. Califano D, Pignata S, Losito NS, Ottaiano A, Greggi S, De Simone V, Cecere S, Aiello C, Esposito F, Fusco A and Chiappetta G: High HMGA2 expression and high body mass index negatively affect the prognosis of patients with ovarian cancer. J Cell Physiol 229: 53-59, 2014.

7. Bi X, Rexer B, Arteaga CL, Guo M and Mahadevan-Jansen A: Evaluating HER2 amplification status and acquired drug resistance in breast cancer cells using Raman spectroscopy. J Biomed Opt 19: 025001, 2014

8. Koochekpour S, Majumdar S, Azabdaftari G, Attwood K, Scioneaux R, Subramani D, Manhardt C, Lorusso GD Willard SS, Thompson H, et al: Serum glutamate levels correlate with gleason score and glutamate blockade decreases proliferation, migration, and invasion and induces apoptosis in prostate cancer cells. Clin Cancer Res 18: 5888-5901, 2012.

9. Jiang L, Xiao L, Sugiura H, Huang X, Ali A, Kuro-o M, Deberardinis RJ and Boothman DA: Metabolic reprogramming during TGF $\beta 1$-induced epithelial-to-mesenchymal transition. Oncogene 34: 3908-3916, 2015.

10. Zadra G, Photopoulos C, Tyekucheva S, Heidari P, Weng QP, Fedele G, Liu H, Scaglia N, Priolo C, Sicinska E, et al: A nove direct activator of AMPK inhibits prostate cancer growth by blocking lipogenesis. EMBO Mol Med 6: 519-538, 2014.

11. Li L, Pilo GM, Li X, Cigliano A, Latte G, Che L, Joseph C, Mela M, Wang C, Jiang L, et al: Inactivation of fatty acid synthase impairs hepatocarcinogenesis driven by AKT in mice and humans. J Hepatol 64: 333-341, 2016.

12. Li J, Dong L, Wei D, Wang X, Zhang S and Li H: Fatty acid synthase mediates the epithelial-mesenchymal transition of breast cancer cells. Int J Biol Sci 10: 171-180, 2014.

13. Grube S, Dünisch P, Frietag D, Klausnitzer M, Sakr Y, Walter J, Kalff R and Ewald C: Overexpression of fatty acid synthase in human gliomas correlates with the WHO tumor grade and inhibition with Orlistat reduced cell viability and triggers apoptosis. J Neurooncol 118: 277-287, 2014.

14. Zaytseva YY, Rychahou PG, Gulhati P, Elliott VA, Mustain WC, O'Connor K, Morris AJ, Sunkara M, Weiss HL, Lee EY and Evers BM: Inhibition of fatty acid synthase attenuates CD44-associated signaling and reduces metastasis in colorectal cancer. Cancer Res 72: 1504-1517, 2012.

15. Duan J, Sun L, Liao W, Wu Z, Wang L and Liao W: Overexpression of fatty acid synthase predicts a poor prognosis for human gastric cancer. Mol Med Rep 13: 3027-3035, 2016.

16. Hou W, Fei M, Qin CY, Zhu X, Greshock J, Liu P, Zhou Y, Wang H, Ye BC and Qin CY: High overexpression of fatty acid synthase is associated with poor survival in Chinese patients with gastric carcinoma. Exp Ther Med 4: 999-1004, 2012.

17. Ito T, Sato K, Maekawa H, Sakurada M, Orita H, Shimada K, Daida H, Wada R, Abe M, Hino O and Kajiyama Y: Elevated levels of serum fatty acid synthase in patients with gastric carcinoma. Oncol Lett 7: 616-620, 2014.

18. Gonzalez DM and Medici D: Signaling mechanisms of the epithelial-mesenchymal transition. Sci Signal 7: re8, 2014.
19. Tania M, Khan MA and Fu J: Epithelial to mesenchymal transition inducing transcription factors and metastatic cancer. Tumour Biol 35: 7335-7342, 2014.

20. Taipale $\mathrm{J}$ and Beachy PA: The Hedgehog and Wntsignalling pathways in cancer. Nature 411: 349-354, 2001

21. Fukaya M, Isohata N, Nakanishi Y, Aoyagi K, Ochiya T, Saeki N, Yanagihara K, Nakanishi Y, Taniguchi $\mathrm{H}$, Sakamoto H, et al: Hedgehog signal activation in gastric pit cell and in diffuse-type gastric cancer. Gastroenterology 131: 14-29, 2006.

22. Chong Y, Tang D, Xiong Q, Jiang X, Xu C, Huang Y, Wang J, Zhou H, Shi Y, Wu X and Wang D: Galectin-1 from cancer-associated fibroblasts induces epithelial-mesenchymal transition through $\beta 1$ integrin-mediated upregulation of Gli1 in gastric cancer. J Exp Clin Cancer Res 35: 175, 2016.

23. Wang ZS, Shen Y, Li X, Zhou CZ, Wen YG, Jin YB and Li JK: Significance and prognostic value of Gli-1 and Snail/E-cadherin expression in progressive gastric cancer. Tumour Biol 35: 1357-1363, 2014.

24. Yoo YA, Kang MH, Lee HJ, Kim BH, Park JK, Kim HK, Kim JS and Oh SC: Sonic hedgehog pathway promotes metastasis and lymphangiogenesis via activation of Akt, EMT, and MMP-9 pathway in gastric cancer. Cancer Res 71: 7061-7070, 2011.

25. Livak KJ and Schmittgen TD: Analysis of relative gene expression data using real-time quantitative PCR and the 2(-delta delta C(T)) method. Methods 25: 402-408, 2001

26. Grunt TW, Wagner R, Grusch M, Berger W, Singer CF, Marian B, Zielinski CC and Lupu R: Interaction between fatty acid synthase and ErbB systems in ovarian cancer cells. Biochem Biophys Res Commun 385: 454-459, 2009.

27. Tomek K, Wagner R, Varga F, Singer CF, Karlic H and Grunt TW: Blockade of fatty acid synthase induces ubiquitination and degradation of phosphatidylinositol-3-kinase signaling proteins in ovarian cancer. Mol Cancer Res 9: 1767-1779, 2011.

28. Wagner R, Stübiger G, Veigel D, Wuczkowski M, Lanzerstorfer P, Weghuber J, Karteris E, Nowikovsky K, Wilfinger-Lutz N, Singer CF, et al: Multi-level suppression of receptor-PI3K-mTORC1 by fatty acid synthase inhibitors is crucial for their efficacy against ovarian cancer cells. Oncotarget 8: 11600-11613, 2017

29. Calvisi DF, Wang C, Ho C, Ladu S, Lee SA, Mattu S, Destefanis G, Delogu S,Zimmermann A, Ericsson J, et al: Increased lipogenesis, induced by AKT-mTORC1-RPS6 signaling, promotes development of human hepatocellular carcinoma. Gastroenterology 140: 1071-1083, 2011

30. Zheng SS, Gao JG, Liu ZJ, Zhang XH, Wu S, Weng BW, Wang YL, Hou SC and Jiang B: Downregulation of fatty acid synthase complex suppressed cell migration by targeting phosphor-AKT in bladder cancer. Mol Med Rep 13: 1845-1850, 2016.

31. Chang L, Wu P, Senthilkumar R, Tian X, Liu H, Shen X, Tao Z and Huang P: Loss of fatty acid synthase suppresses the malignant phenotype of colorectal cancer cells by down-regulating energy metabolism and mTOR signaling pathway. J Cancer Res Clin Oncol 142: 59-72, 2016.

32. Shimobayashi $M$ and Hall MN: Making new contacts: The mTOR network in metabolism and signalling crosstalk. Nat Rev Mol Cell Biol 15: 155-162, 2014.

33. Teperino R, Aberger F, Esterbauer H, Riobo N and Pospisilik JA: Canonical and non-canonical Hedgehog signalling and the control of metabolism. Semin Cell Dev Biol 33: 81-92, 2014.

34. Wang Y, Ding Q, Yen CJ, Xia W, Izzo JG, Lang JY, Li CW, Hsu JL, Miller SA, Wang X, et al: The crosstalk of mTOR/S6K1 and Hedgehog pathways. Cancer Cell 21: 374-387, 2012.

35. Furuta E, Pai SK, Zhan R, Bandyopadhyay S, Watabe M, Mo YY, Hirota S, Hosobe S, Tsukada T, Miura K, et al: Fatty acid synthase gene is upregulated by hypoxia via activation of Akt and sterol regulatory element binding protein-1. Cancer Res 68: 1003-1011, 2008.

36. Bhatia B, Hsieh M, Kenney AM and Nahlé Z: Mitogenic sonic hedgehog signaling drives E2F1-dependent lipogenesis in progenitor cells and Medulloblastoma. Oncogene 30: 410-422, 2011.

37. Bhatia B, Potts CR, Guldal C, Choi S, Korshunov A, Pfister S, Kenney AM and Nahlé ZA: Hedgehog-mediated regulation of PPAR $\gamma$ controls metabolic patterns in neural precursors and shh-driven medulloblastoma. Acta Neuropath 123: 587-600, 2012. 